\title{
Three Patient Kindred with a Novel Phenotype of Osteogenesis Imperfecta due to a COL1A1 Variant
}

\author{
(1) Nidhi Gupta1,2,*, (1) Seth W. Gregory33, (1) David R. Deyle4, (1) Peter J. Tebben2,5,* \\ 1 Vanderbilt University Medical Center, Department of Pediatrics, Division of Pediatric Endocrinology and Diabetes, Tennessee, USA \\ 2 Mayo Clinic, Department of Pediatric and Adolescent Medicine, Division of Endocrinology and Metabolism, Minnesota, USA \\ 3 Mayo Clinic Health System, Department of Pediatric and Adolescent Medicine, Minnesota, USA \\ ${ }^{4}$ Mayo Clinic, Department of Medical Genetics, Minnesota, USA \\ ${ }^{5}$ Mayo Clinic, Department of Medicine, Division of Endocrinology, Metabolism and Nutrition, Minnesota, USA \\ *Corresponding authors
}

\begin{abstract}
What is already known on this topic?
Osteogenesis imperfecta (OI) is a heterogeneous connective tissue disorder characterized by reduced bone mass and increased bone fragility. Peak fracture rates in OI occur during the toddler and adolescent years, decline during adulthood, and increase again after age 55 years.
\end{abstract}

\section{What this study adds?}

We describe a kindred including three members who presented with a unique phenotype of OI, presumably due to a proline-to-leucine missense variant in the COL1A1 gene. All three members had a pattern of prenatal bone deformities, followed by multiple, nontraumatic long bone fractures within the first two years of life and then an absence of nontraumatic fractures thereafter. To our knowledge, a clinical phenotype of OI characterized by cessation of nontraumatic fractures after the first two years of life has not been described previously.

\begin{abstract}
Osteogenesis imperfecta (OI) is characterized by fractures and progressive bone deformities. Fracture rates peak during the toddler and adolescent years and decline during adulthood but do not stop entirely. We describe a kindred, the affected members of which were the mother and two sons, who presented with an apparently unique phenotype of OI. Our patients demonstrated a pattern of prenatal bone deformities followed by multiple, nontraumatic long bone fractures within the first two years of life and then an absence of nontraumatic fractures thereafter. No extra-skeletal manifestations have been noted to date. The mother did not receive bisphosphonate therapy but had no nontraumatic fractures after the age of five months. Intravenous bisphosphonate therapy was started for both sons within two months of birth, with the most recent infusions at age 18 months and 28 months in Patients 2 and 3, respectively. Two patients harbored a variant of uncertain significance in the COL1A1 gene. This heterozygous variant, c.3548C > T; p. (Pro1183Leu), is listed in the OI Variant Database as affecting only one other individual with osteopenia. We describe three family members with a unique presenting phenotype of OI, characterized by cessation of nontraumatic fractures after the first two years of life.
\end{abstract}

Keywords: Fragility fractures, collagen, child, bisphosphonates, bone density

\section{Introduction}

Osteogenesis imperfecta (OI) is a heterogeneous connective tissue disorder characterized by reduced bone mass and increased bone fragility $(1,2)$. The disorder is primarily caused by variants in the genes involved in the synthesis or post-transcription modification of type 1 collagen (3). Type

Presented in: Poster presentation at the 2018 Annual Meeting of the American Society for Bone and Mineral Research, Montreal, Canada, October 2018.

Address for Correspondence: Peter J. Tebben MD, Mayo Clinic College of Medicine, Departments of Pediatric and Adolescent Medicine and Internal Medicine, Division of Endocrinology and Metabolism, Minnesota, USA Phone: +9507-284-3300 E-mail: tebben.peter@mayo.edu ORCID: orcid.org/0000-0002-2147-0891

Address for Correspondence: Nidhi Gupta MD, Vanderbilt University Medical Center, Department of Pediatrics, Division of Pediatric Endocrinology and Diabetes, Tennessee, USA

Phone: +615-343-0794 E-mail: nidhi.gupta@vumc.org ORCID: orcid.org/0000-0001-6485-3318

'Copyright 2021 by Turkish Pediatric Endocrinology and Diabetes Society

The Journal of Clinical Research in Pediatric Endocrinology published by Galenos Publishing House.
Conflict of interest: None declared Received: 28.01.2020 Accepted: 19.05.2020 
1 procollagen is composed of two proa1(I) and one proa2(I) chains that are encoded by the COL1A1 (OMIM \#120150) and COL1A2 (OMIM \#120160) genes, respectively (2). The triple-stranded procollagen molecule is assembled and then secreted into the extracellular space, where the propeptides are enzymatically cleaved to form mature collagen (4). Although there are numerous other Ol-related genes, most cases of OI (85-90\%) are caused by variants in the COL1A1 and COL1A2 genes.

Typically, point variants affecting glycine residues in the triple-helix structure of type 1 collagen have been predicted to disrupt protein folding, resulting in OI (5). The substitution of leucine for proline (Pro) in exon 49 of the COL1A1 gene is listed in the OI Variant Database but to date, it has been associated with only one individual affected by osteopenia and it has been designated as a variant of unknown pathogenicity (6).

The severity of OI varies from mild, to progressively deforming, to perinatally lethal (7). Peak fracture rates in OI occur during the toddler and adolescent years, decline during adulthood, and increase again after age 55 years (8). However, to our knowledge, a clinical phenotype of OI characterized by cessation of nontraumatic fractures after the first two years of life has not been described previously.

We describe a kindred of three members who presented with a unique phenotype of OI, presumably due to a Proto-leucine missense variant in the COL1A1 gene. All three members had a pattern of prenatal bone deformities followed by multiple, nontraumatic, long bone fractures within the first two years of life and then an absence of nontraumatic fractures thereafter.

\section{Case Reports}

We describe a nonconsanguineous family of European descent, in which three members were affected by OI (Table 1, Figure 1). Informed verbal consent was obtained from the mother

\section{Patient 1: Mother}

The mother received the diagnosis of OI when she was 23 years old, after the birth of her second child (Patient 2), whose prenatal ultrasound suggested OI. The mother had nine nontraumatic long bone fractures, primarily femoral, before the age of five months. Her prenatal ultrasound findings were unknown. She did not receive bisphosphonate treatment but did not have any other nontraumatic fractures after five months of age. She had normal sclerae, dentition, hearing, echocardiogram and stature $(159.8 \mathrm{~cm}$, $25^{\text {th }}$ percentile) (9). She had no spinal or cranial deformities, no joint hypermobility and did not bruise easily. We were unable to obtain data regarding her bone turnover markers and bone mineral density (BMD). She had an unaffected 17-year-old daughter. As of manuscript writing, Patient 1 was 39 years old.

\section{Patient 2: Older Sibling}

Patient 2 was the second child of Patient 1 and an unaffected father. The father's height was $188 \mathrm{~cm}$ ( $94^{\text {th }}$ percentile) (9). At five months gestation, a prenatal ultrasound showed evidence of bowing of both femurs. Patient 2 was born at 38 weeks gestation via caesarean delivery without respiratory distress at birth. His birth weight was $4.28 \mathrm{~kg}\left(90^{\text {th }}\right.$ percentile), length was $52 \mathrm{~cm}\left(50^{\text {th }}\right.$ percentile) and head circumference was $38 \mathrm{~cm}$ ( $90^{\text {th }}$ percentile) (9). At age three days, during circumcision, he had a right femur fracture (Figure 1). Subsequently, he had fractures of bilateral humeri and femurs at six days of life and sustained a fracture of the left femur at age four weeks. There was minimal or no trauma associated with any of these fractures.

A diagnosis of OI was considered and pamidronate infusions were initiated $(0.75 \mathrm{mg} / \mathrm{kg}$ per day on two consecutive days, every two months with a total of eight infusions) from the age of six weeks through 18 months at another center. He tolerated the infusions well. Age-related developmental milestones were normal. At age three months, he was at the $95^{\text {th }}$ percentile for length and $97^{\text {th }}$ percentile for weight. His sclerae, hearing and dentition were normal. He had no spinal or cranial deformities, no joint hypermobility, and did not bruise easily. At age 14 months, his lumbar spine BMD was $0.51 \mathrm{~g} / \mathrm{cm}^{2}$ and left hip BMD was $0.57 \mathrm{~g} / \mathrm{cm}^{2}$ (reference range not available for this age group) (10). He had normal serum calcium (10.6 mg/dL; reference range 9-11 mg/dL) and total alkaline phosphatase $(274 \mathrm{U} / \mathrm{L}$; reference range 150-420 U/L).

Given the paucity of fractures (no fractures since age four weeks, despite being very active), pamidronate therapy was discontinued at age 18 months. As of manuscript writing, he was 17 years old, had normal ambulation and no deformities. A follow-up BMD was not available for him. His height was at the $75^{\text {th }}$ percentile and weight was at the $95^{\text {th }}$ percentile. His corrected midparental height was at the $65^{\text {th }}$ percentile.

\section{Patient 3: Younger Sibling}

Patient 3 was born at 39 weeks gestation to Patient 1 and a different father. The father's height was $170 \mathrm{~cm}$ ( $17^{\text {th }}$ percentile) (9). At 26 weeks gestation, an ultrasound showed bowing of both femurs. Patient 3 was born via caesarean delivery, without respiratory distress at birth. 
Table 1. Clinical characteristics of kindred cases

\begin{tabular}{|c|c|c|c|}
\hline & Patient 1 & Patient 2 & Patient 3 \\
\hline & Mother & Older sibling & Younger sibling \\
\hline Current age & $39 y$ & $17 \mathrm{y}$ & $36 \mathrm{~m}$ \\
\hline Current height (percentile) & 25 & 75 & 27 \\
\hline Fractures, age & $\begin{array}{l}\text { R femur, }<5 \mathrm{~m} \\
\text { L femur, }<5 \mathrm{~m}\end{array}$ & $\begin{array}{l}\text { R femur, } 3 \mathrm{~d} \\
\text { R humerus, } 6 \mathrm{~d} \\
\text { L humerus, } 6 \mathrm{~d} \\
\text { R femur, } 6 \mathrm{~d} \\
\text { L femur, } 6 \mathrm{~d} \\
\text { L femur, } 4 \mathrm{w}\end{array}$ & $\begin{array}{l}\mathrm{L} 9^{\text {th }} \text { rib, } 1 \mathrm{~d} \\
\mathrm{R} 8-10^{\text {th }} \text { rib, } 2 \mathrm{w} \\
\mathrm{R} \text { tibia, } 5 \mathrm{w} \\
\mathrm{R} \text { femur, } 5 \mathrm{w} \\
\mathrm{L} \text { femur, } 5 \mathrm{w} \\
\text { R femur, } 6 \mathrm{w} \\
\mathrm{L} \text { femur, } 6 \mathrm{w} \\
\mathrm{L} \text { tibia, } 15 \mathrm{w} \\
\mathrm{L} 5 \text { compression, } 21 \mathrm{~m}\end{array}$ \\
\hline Total fractures, $n$ & 9 & 6 & 11 \\
\hline $\operatorname{BMD}\left(\mathrm{g} / \mathrm{cm}^{2}\right)$, age & N/A & $\begin{array}{l}14 \mathrm{~m} \\
\text { Lumbar spine: } 0.51 \\
\text { L hip: } 0.57\end{array}$ & $\begin{array}{l}\mathbf{1 2} \mathbf{~ m} \\
\text { Lumbar spine: } 0.44 \\
\text { TBLH: } 0.34 \\
\mathbf{2 8 ~ m} \\
\text { Lumbar spine: } 0.54 \\
\text { TBLH: } 0.46\end{array}$ \\
\hline Bisphosphonate treatment & No & $\begin{array}{l}\text { Yes } \\
\text { Pamidronate }\end{array}$ & $\begin{array}{l}\text { Yes } \\
\text { Zoledronic acid }\end{array}$ \\
\hline Total infusions, $\mathrm{n}$ & - & 8 & 6 \\
\hline Age at last infusion & - & $18 \mathrm{~m}$ & $28 \mathrm{~m}$ \\
\hline Sclerae & Normal & Normal & Normal \\
\hline Dentition & Normal & Normal & Normal \\
\hline Hearing & Normal & Normal & Normal \\
\hline Echocardiogram & Normal & N/A & N/A \\
\hline Spinal deformity & None & None & None \\
\hline Extremity deformity & None & None & None \\
\hline Joint mobility & Normal & Normal & Normal \\
\hline Easy bruising & None & None & None \\
\hline Genotype & $\begin{array}{l}\text { c. } 3548 C>\text { T; p.(Pro11 83Leu) } \\
\text { Heterozygous }\end{array}$ & $\begin{array}{l}\text { c. } 3548 \mathrm{C}>\mathrm{T} ; \text { p. }(\text { Pro } 1183 \mathrm{Leu}) \\
\text { Heterozygous }\end{array}$ & N/A \\
\hline
\end{tabular}

His birth weight was $3.8 \mathrm{~kg}$ ( $75^{\text {th }}$ percentile), length was 48 $\mathrm{cm}$ (25 $5^{\text {th }}$ percentile) and head circumference was $36.5 \mathrm{~cm}$ (65 $5^{\text {th }}$ percentile). Radiographic studies on the first day of life confirmed left posterior ninth rib fracture and femoral bowing bilaterally (Figure 2). Wormian bones were noted along the lambdoid sutures. He had nontraumatic fractures of the eighth through tenth ribs on the right side at two weeks, right tibia and bilateral femur fractures at five weeks, bilateral femur fractures at six weeks (distinct locations each time) and left tibia fracture at 15 weeks.

Physical examination showed that he had white sclerae and no dysmorphic features. His hearing screen was normal.
Serum ionized calcium $(5.89 \mathrm{mg} / \mathrm{dL}$; reference range 3.9$6.0 \mathrm{mg} / \mathrm{dL}$ ), phosphorus $(6.3 \mathrm{mg} / \mathrm{dL}$; reference range 4.5 $9.0 \mathrm{mg} / \mathrm{dL}$ ), total alkaline phosphatase (250 U/L; reference range 150-420 U/L) and 25-hydroxy-vitamin D (37 ng/mL; reference range $\geq 20 \mathrm{ng} / \mathrm{mL}$ ) concentrations were normal. Zoledronic acid infusions were initiated every three months from age three weeks to nine months $(0.0125 \mathrm{mg} / \mathrm{kg}$ for the first dose, $0.025 \mathrm{mg} / \mathrm{kg}$ for the next three doses, each infused over 60 minutes). The fracture at age 15 weeks occurred after he had received two infusions of zoledronic acid. Given the paucity of further symptoms of OI and the fact that his mother and older sibling had no fractures after 
A
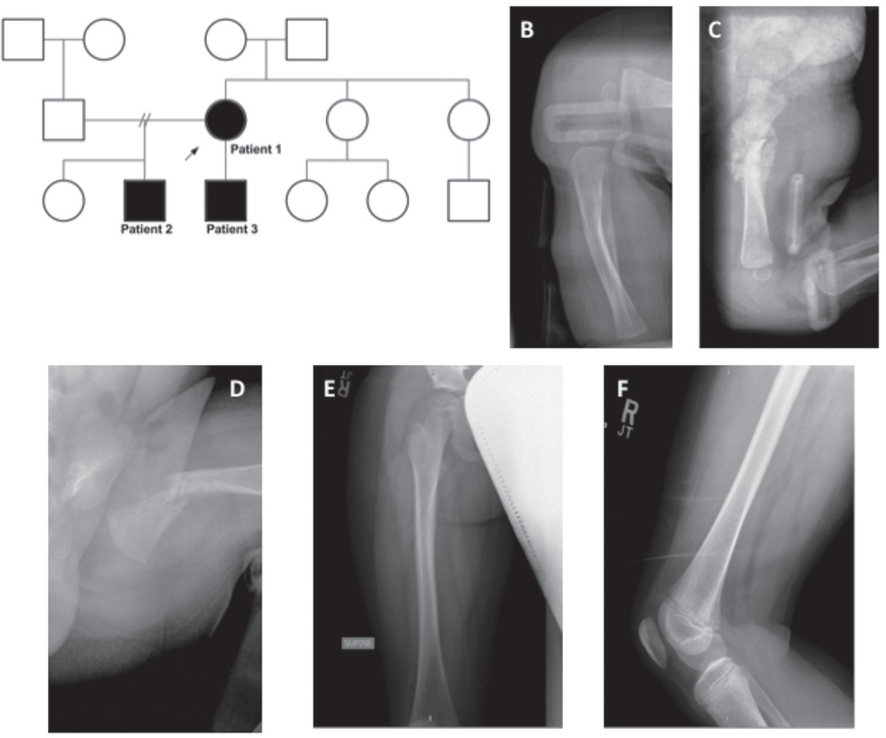

Figure 1. (A) Pedigree chart. The index case is indicated by the arrow. (B-F) Radiographs of Patient 2. (B) Bowing of midright tibial shaft at age three weeks, lateral view. (C) Abundant callus formation around a healing midshaft right femoral fracture at age three weeks, lateral view. (D) Acute proximal-shaft left femoral fracture at age four weeks, anteroposterior view. (E) Right femur anteroposterior view at age 10 years. (F) Right femur lateral view at age 10 years
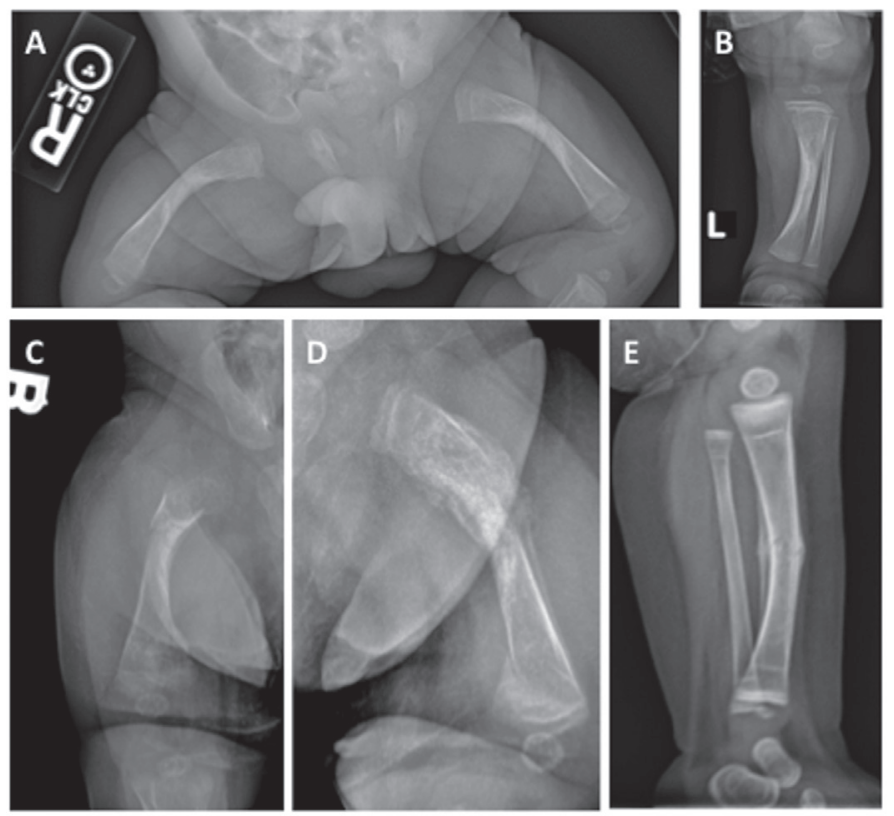

Figure 2. Radiographs of Patient 3. (A) Proximal femoral shaft bowing, right greater than the left, at age two weeks, anteroposterior view. Note the mild diffuse osteopenia. (B) Left tibial midshaft bowing at age two weeks, anteroposterior view. (C) Proximal right femoral fracture at age six weeks, lateral view. (D) Periosteal reaction and callus formation around a healing proximal left femoral fracture sustained at age six weeks, lateral view. (E) Transverse midshaft fracture of left tibia at age 15 weeks, lateral view the first few months of life, bisphosphonate therapy was discontinued when he was nine months old.

At age 21 months, he was noted to have a new mild compression of the anterior-superior endplate of the $5^{\text {th }}$ lumbar vertebral body (L5). He also had a mildly displaced oblique fracture of left distal tibial metaphysis due to moderate trauma (his leg was caught on the edge of a slide while sitting on his father's lap) at age 25 months. He was treated with two additional infusions of zoledronic acid at 21 months and 28 months (a total of six infusions). He has not sustained additional low-trauma fractures since. The mild L5 vertebral body deformity was less apparent on subsequent imaging seven months later.

He achieved normal developmental milestones, including appropriate dentition for age. Baseline BMD was obtained at 12 months (lumbar spine $0.44 \mathrm{~g} / \mathrm{cm}^{2}$; total body excluding head $0.34 \mathrm{~g} / \mathrm{cm}^{2}$; reference range not available for this age group) (10). A follow-up BMD at age 28 months after six zoledronic acid infusions revealed an improvement of $23.7 \%$ in spinal bone density $\left(0.54 \mathrm{~g} / \mathrm{cm}^{2}\right)$ and $36.5 \%$ in total body, excluding head, bone density $\left(0.46 \mathrm{~g} / \mathrm{cm}^{2}\right)$, compared to baseline. As of manuscript writing, he was 36 months old and at $27^{\text {th }}$ percentile for length and $81^{\text {st }}$ percentile for weight. His corrected midparental height was at the $20^{\text {th }}$ percentile.

\section{DNA Sequencing and in silico Analysis}

Clinical information was collected from the patients and abstracted from the medical records. Conformationsensitive gel electrophoresis (CSGE) was performed by Matrix DNA Diagnosis (New Orleans, LA, USA). The variant was described using the Human Genome Variation Society nomenclature (11). The pathogenicity of the identified variant was analyzed using the in silico prediction software, Alamut Visual (missense predictors: Align GVGD v.2007, SIFT v.6.20 and PolyPhen-2 HumVar; splicing algorithms: SpliceSiteFinder-like, MaxEntScan, NNSPLICE, GeneSplicer and HSF) (12).

\section{Results}

\section{Identification of the COL1A1 Variant}

Given the concern for OI in Patient 2, CSGE was performed for the COL1A1- and COL1A2- coding exons. The CSGE analysis identified a heterozygous missense alteration in exon 49 of the COL1A1 gene resulting in a $\mathrm{C}$ to $\mathrm{T}$ nucleotide substitution that converted a Pro (CCT) to a leucine (CTT), [NM_000088.3:c.3548C > T; p.(Pro1183Leu), (RefSeqGene NG_007400.1:g.19734C > T)]. 
The patient's mother was heterozygous for the same variant and no variations were present in the father. The variant was classified as 'variant of uncertain significance'. In silico analysis was equivocal for potential pathogenicity of this variant (Align GVGD - Class C65 deleterious; SIFT deleterious; PolyPhen-2 - benign). No significant changes were noted in canonical splice sites.

\section{Discussion}

We describe a kindred with a unique presenting phenotype of OI. The OI was presumably attributable to a missense variant c.3548C > T; p.(Pro1183Leu) that replaced Pro with leucine in the $\mathrm{Y}$ position of the glycine-Xaa-Yaa sequence of the proa1(I) chain (exon 49 of COL1A1). The same variant has been reported previously in only one individual [c.3548C > T; p.(Pro1183Leu)] (6). The referral reason for testing that individual was reported as symptoms of OI and a strong family history of recurrent femoral fractures and osteopenia. The variant was designated to be of unknown pathogenicity, and further family studies to clarify pathogenicity were requested. As of manuscript writing, that patient was 49 years old, and updates on the patient and results from family studies could not be obtained (personal communication, Dr. Meena Balasubramanian, 2018). As more individuals with this specific variant are identified, the clinical phenotype of this form of OI will become clearer.

Lim et al (13) described a patient with Ser40Trp variant in IFITM5 gene, who presented with multiple fractures in the prenatal period. She remained fracture free after birth with normal BMD. She had blue sclerae, progressive lower limb deformities during childhood and severe short stature (standard deviation -3.5). Her mother, who did not have a history of fractures, was noted to have somatogonadal mosaicism for this variant and became pregnant with a second child with multiple prenatal fractures, found to have the same variant. This is in contrast with our patients who had normal sclerae, absence of postnatal limb deformities, normal stature and a variant in the COL1A1 gene, although with similar phenotypic bone deformity pattern.

Type 1 procollagen helical domains contain a repetitive glycine-Xaa-Yaa sequence, where Xaa and Yaa are often the amino acids Pro or hydroxyproline (Hyp) $(14,15)$. Some evidence suggests that Pro and Hyp are responsible for the thermal stability of the triple-helix structure (16). Bryan et al (17) hypothesized that a missense variant in the highly stable region of the collagen molecule, such as Gly-ProHyp, will lead to a greater disruption and more severe clinical consequences than the same missense variant in a less stable region. Collagen chains with these abnormal molecules are overmodified and secreted inefficiently, which disrupts helix stability (18).

Various factors have been proposed to explain how the substitution of one amino acid for another affects collagen processing and the phenotype of $\mathrm{OI}$, including the coordinates of the variant and its position relative to the C-terminus of the propeptide $(5,14,19)$. The proximity of a variant to the C-terminus of the molecule is important because assembly of the collagen triple-helix chain begins at the $\mathrm{C}$-terminal end and propagates toward the $\mathrm{N}$-terminal end (20). Our kindred harbored a variant close to the C-terminus, at amino acid position 1183 on exon 49 of the COL1A1 gene. Exon 49 is 283 base pairs long (CDNA base numbers 3532-3814) and encodes the last 15 amino acids of the triple-helix region (amino acids 1178-1192), the entire C-telopeptide (amino acids 1193-1218) and part of the C-propeptide domain (amino acids 1219-1271). A small number of point variants in the C-terminal propeptide have been identified as causing varying clinical phenotypes of OI, ranging from mild to lethal and high bone-mass OI $(18,19,21)$. Although missense variants in the C-propeptide are known to impair or prevent prod chain assembly in the endoplasmic reticulum (19), the outcome of each substitution may differ, depending on the specific gene transcript.

For patients with milder forms of OI, the incidence of fracture declines with age but does not stop entirely (8). Interestingly, cessation of OI associated fractures after the first two years of life, as seen in our patients, has not been described in the literature in association with COL1A1 variants. The pathophysiology resulting in this phenotype remains uncertain. There is evidence that posttranslational modification of procollagen is temporally regulated, and this regulation may be crucial for its folding, secretion, and extracellular matrix assembly (22). We speculate that a sequential improvement in these processes during the first two years of life in our patients resulted in paucity of fractures thereafter.

Finally, the long-term benefit of intravenous bisphosphonate therapy in our patients remains unclear, particularly because Patient 1 did not receive this therapy and did not sustain any nontraumatic fractures after five months of age. Patient 2 received his last infusion of pamidronate at age 18 months. At age 17 years, he was of normal adult height and remained fracture free, despite an unrestricted, active lifestyle. We speculate that prolonged bisphosphonate therapy may not provide additional benefit to those with this form of OI, although our sample size and duration of follow-up is too small to draw a definitive conclusion. 
Our case series has certain other limitations including lack of genetic testing for variants in other OI-related genes such as IFITM5, which have been associated with a phenotype similar to that seen in our patients. Patient 1 and Patient 2 underwent genetic testing almost 17 years ago. To our knowledge, at that point in time, typical OI genetic testing included primarily COL1A1 and COL1A2 genes. Currently, we do not have the funding available to update genetic testing for Patient 1 and Patient 2, or send genetic testing for Patient 3 for research purposes. It would have entailed a huge economic burden on the family and the genetic testing results were unlikely to influence clinical decision making.

\section{Conclusion}

We describe a three-patient kindred with a unique phenotype of OI, presumably due to a variant in the COL1A1 gene. The OI phenotype of affected individuals included a pattern of mild-moderate bone deformities prenatally and multiple nontraumatic fractures limited to the first two years of life. We speculate that the Pro-to-leucine substitution in close proximity to the C-propeptide domain might have influenced folding of the triple helix or helix stability. Given the small number of patients in our kindred, the full range of phenotypes associated with this variant remains to be established. Whether antiresorptive therapy is beneficial later in life also remains unclear.

\section{Acknowledgement}

We thank the family for their participation in this report. We thank Christine Koellner, MS, LCGC and Nikita Mehta, MS, L/CGC, Department of Laboratory Medicine and Pathology, Mayo Clinic for their help with the in silico analysis. We thank Dr. Meena Balasubramanian for sharing information about the patient at Sheffield Children's NHS Foundation Trust, UK.

\section{Ethics}

Informed Consent: Informed verbal consent was obtained from the mother.

Peer-review: Externally and internally peer-reviewed.

\section{Authorship Contributions}

Surgical and Medical Practices: Nidhi Gupta, Seth W. Gregory, David R. Deyle, Peter J. Tebben, Concept: Nidhi Gupta, Seth W. Gregory, David R. Deyle, Peter J. Tebben, Design: Nidhi Gupta, Seth W. Gregory, David R. Deyle, Peter J. Tebben, Data Collection or Processing: Nidhi Gupta, Seth W. Gregory, David R. Deyle, Peter J. Tebben, Analysis or Interpretation: Nidhi Gupta, Seth W. Gregory, David R. Deyle, Peter J.
Tebben, Literature Search: Nidhi Gupta, Seth W. Gregory, David R. Deyle, Peter J. Tebben, Writing: Nidhi Gupta, Seth W. Gregory, David R. Deyle, Peter J. Tebben.

Financial Disclosure: The authors declared that this study received no financial support.

\section{References}

1. Pollitt RC, Saraff V, Dalton A, Webb EA, Shaw NJ, Sobey GJ, Mughal MZ, Hobson E, Ali F, Bishop NJ, Arundel P, Högler W, Balasubramanian M. Phenotypic variability in patients with osteogenesis imperfecta caused by BMP1 mutations. Am J Med Genet A 2016;170:3150-3156. Epub 2016 Aug 30

2. Marini JC, Forlino A, Cabral WA, Barnes AM, San Antonio JD, Milgrom S, Hyland JC, Körkkö J, Prockop DJ, De Paepe A, Coucke P, Symoens S, Glorieux FH, Roughley PJ, Lund AM, Kuurila-Svahn K, Hartikka H, Cohn DH, Krakow D, Mottes M, Schwarze U, Chen D, Yang K, Kuslich C, Troendle J, Dalgleish R, Byers PH. Consortium for osteogenesis imperfecta mutations in the helical domain of type I collagen: regions rich in lethal mutations align with collagen binding sites for integrins and proteoglycans. Hum Mutat 2007;28:209-221.

3. Marini JC, Blissett AR. New genes in bone development: what's new in osteogenesis imperfecta. J Clin Endocrinol Metab 2013;98:3095-3103. Epub 2013 Jun 1

4. Pace JM, Atkinson M, Willing MC, Wallis G, Byers PH. Deletions and duplications of Gly-Xaa-Yaa triplet repeats in the triple helical domains of type I collagen chains disrupt helix formation and result in several types of osteogenesis imperfecta. Hum Mutat 2001;18:319-326.

5. Pollitt R, McMahon R, Nunn J, Bamford R, Afifi A, Bishop N, Dalton A. Mutation analysis of COL1A1 and COL1A2 in patients diagnosed with osteogenesis imperfecta type I-IV. Hum Mutat 2006;27:716.

6. OI Database. Osteogenesis Imperfecta Variant Database COL1A1. Leiden University, Medical Center, 2018.

7. Van Dijk FS, Sillence DO. Osteogenesis imperfecta: clinical diagnosis, nomenclature and severity assessment. Am J Med Genet A 2014;164:1470-1481. Epub 2014 Apr 8

8. Folkestad L, Hald JD, Ersbøll AK, Gram J, Hermann AP, Langdahl B, Abrahamsen B, Brixen K. Fracture Rates and Fracture Sites in Patients With Osteogenesis Imperfecta: A Nationwide Register-Based Cohort Study. J Bone Miner Res 2017;32:125-134. Epub 2016 Aug 29

9. Clinical Growth Charts. Centers for Disease Control and Prevention. Last accessed date: April 2021. Available from: https://www.cdc.gov/ growthcharts/clinical_charts.htm

10. van der Sluis IM, de Ridder MA, Boot AM, Krenning EP, de Muinck Keizer-Schrama SM. Reference data for bone density and body composition measured with dual energy $\mathrm{x}$ ray absorptiometry in white children and young adults. Arch Dis Child 2002;87:341-347.

11. den Dunnen JT, Dalgleish R, Maglott DR, Hart RK, Greenblatt MS, McGowan-Jordan J, Roux AF, Smith T, Antonarakis SE, Taschner PE. HGVS Recommendations for the Description of Sequence Variants: 2016 Update. Hum Mutat 2016;37:564-569. Epub 2016 Mar 25

12. Alamut Visual: A mutation analysis software. Last accessed date: April 2021. Available from: https://www.interactive-biosoftware.com/alamutvisual/

13. Lim JY, Bhatia NS, Vasanwala RF, Chay PL, Lim KBL, Khoo PC, Schwarze U, Jamuar SS. A novel Ser40Trp variant in IFITM5 in a family with osteogenesis imperfecta and review of the literature. Clin Dysmorphol 2019;28:120-125. 
14. Xiao J, Yang Z, Sun X, Addabbo R, Baum J. Local amino acid sequence patterns dominate the heterogeneous phenotype for the collagen connective tissue disease Osteogenesis Imperfecta resulting from Gly mutations. J Struct Biol 2015;192:127-137. Epub 2015 May 15

15. Brodsky B, Thiagarajan G, Madhan B, Kar K. Triple-helical peptides: an approach to collagen conformation, stability, and self-association. Biopolymers 2008;89:345-353.

16. Nishi Y, Uchiyama S, Doi M, Nishiuchi Y, Nakazawa T, Ohkubo T, Kobayashi Y. Different effects of 4-hydroxyproline and 4-fluoroproline on the stability of collagen triple helix. Biochemistry 2005;44:60346042 .

17. Bryan MA, Cheng H, Brodsky B. Sequence environment of mutation affects stability and folding in collagen model peptides of osteogenesis imperfecta. Biopolymers 2011;96:4-13.
18. Byers PH, Wallis GA, Willing MC. Osteogenesis imperfecta: translation of mutation to phenotype. J Med Genet 1991;28:433-442.

19. Chessler SD, Wallis GA, Byers PH. Mutations in the carboxyl-terminal propeptide of the pro alpha 1(I) chain of type I collagen result in defective chain association and produce lethal osteogenesis imperfecta. J Biol Chem 1993;268:18218-18225.

20. Dalgleish R. The human type I collagen mutation database. Nucleic Acids Res 1997;25:181-187.

21. Symoens S, Hulmes DJ, Bourhis JM, Coucke PJ, De Paepe A, Malfait F. Type I procollagen C-propeptide defects: study of genotype-phenotype correlation and predictive role of crystal structure. Hum Mutat 2014;35:1330-1341. Epub 2014 Oct 18

22. Forlino A, Marini JC. Osteogenesis imperfecta. Lancet 2016;387:16571671. Epub 2015 Nov 3 